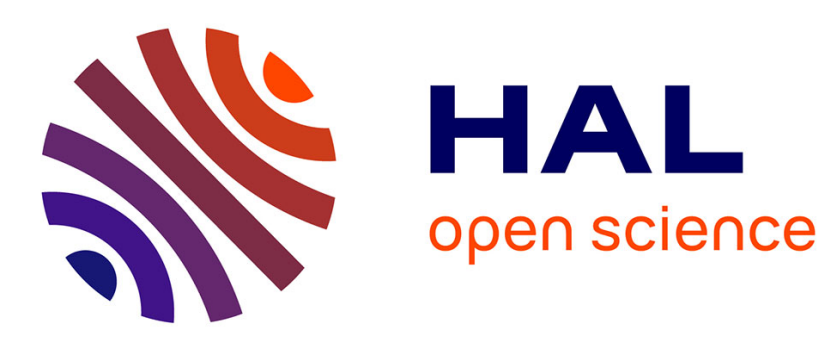

\title{
Pour une approche sociologique de la comptabilité. Réflexions à partir de la réforme comptable chinoise.
}

Corine Eyraud

\section{To cite this version:}

Corine Eyraud. Pour une approche sociologique de la comptabilité. Réflexions à partir de la réforme comptable chinoise.. Sociologie du Travail, 2003, 4, pp.491-508. hal-00154937

\section{HAL Id: hal-00154937 \\ https://hal.science/hal-00154937}

Submitted on 25 Sep 2007

HAL is a multi-disciplinary open access archive for the deposit and dissemination of scientific research documents, whether they are published or not. The documents may come from teaching and research institutions in France or abroad, or from public or private research centers.
L'archive ouverte pluridisciplinaire HAL, est destinée au dépôt et à la diffusion de documents scientifiques de niveau recherche, publiés ou non, émanant des établissements d'enseignement et de recherche français ou étrangers, des laboratoires publics ou privés. 
POUR UNE APPROCHE SOCIOLOGIQUE DE LA

COMPTABILITÉ - RÉFLEXIONS A PARTIR DE LA RÉFORME

COMPTABLE CHINOISE

\author{
Corine EYRAUD \\ Département de Sociologie - LAMES, Université de Provence, \\ 29 avenue R. Schuman \\ 13621 Aix-en-Provence Cédex 1 \\ Tel : 0492501393 \\ eyraud@up.univ-aix.fr
}


La réflexion que nous souhaitons mener ici se situe dans le mouvement de renouveau de la sociologie économique «définie comme la perspective sociologique appliquée aux phénomènes économiques »(Laville, 1997, p.229). Nous nous intéressons plus particulièrement à tout ce qui concerne la comptabilité, notre objectif étant de montrer l'intérêt d'une approche sociologique de ce domaine d'activité ${ }^{1}$. La comptabilité, comme la statistique, est une taxinomie, un système de catégorisations sociales, et c'est sur son caractère construit, sur son historicité que nous voudrions ici mettre l'accent. Pour ce faire, nous nous intéresserons beaucoup plus à la comptabilité normalisée (ou cadres comptables) qu'à la comptabilité pratiquée, pour reprendre la distinction faite par les spécialistes des sciences comptables; et nous analyserons d'une part le système comptable chinois de l'économie planifiée, et d'autre part sa réforme en œuvre depuis 1993. Il s'agit de deux modèles et de deux systèmes très différents ; ils peuvent ainsi nous servir de révélateurs.

\section{Logiques comptable, économique et politique pendant l'économie planifiée $^{2}$}

\footnotetext{
${ }^{1}$ Cet article est le fruit d'une recherche portant sur l'entreprise d'État chinoise ; nous avons passé pour cette recherche dix huit mois en Chine répartis en six séjours de 1993 à 2001, et réalisé une centaine d'entretiens en langue chinoise auprès de directeurs, directeurs financiers et directeurs du personnel d'entreprises d'État, de personnes travaillant dans des services bancaires en relation avec ces entreprises, puis, en plus petit nombre, de chercheurs et enseignants-chercheurs au sein d'instituts de sociologie, économie et comptabilité, et enfin de comptables et officiels en charge des questions comptables.

2 Nos sources, outre les entretiens réalisés, sont essentiellement de langue chinoise; les informations en langue plus accessible sont rares, on peut toutefois se référer à Shanghai University of Finance, 1987, et Tang, 1994.
}

Corine Eyraud. Sociologie du Travail, 2003, n 4, pp. 491-508. 
L'histoire de la comptabilité chinoise ne commence bien évidemment pas avec l'économie planifiée ; la tenue des livres de comptes fut certainement effective dès que les marchands eurent accès à une certaine culture écrite (Goody, 1999) et dans le contexte de développement d'un Etat percevant des impôts. A partir $\mathrm{du} 10^{\text {ème }}$ siècle, une comptabilité complexe se développe utilisant progressivement de nombreux aspects et principes de la partie double, jusqu'à son adoption officielle et sa diffusion pendant la période républicaine (1911-1949). La mise en place du premier plan quinquennal (1953) et d'une logique d'économie planifiée, la transformation des entreprises privées en entreprises d'Etat s'accompagnent de la création d'une importante administration économique et statistique, largement inspirée à ses débuts du (ou des) modèle(s) soviétique(s) (Eyraud, 1999, pp. 60-61). C'est dans ce contexte que vont être élaborés de nouveaux cadres comptables.

\subsection{Quelle comptabilité pour quelle entreprise ?}

Pendant la période de l'économie planifiée, les entreprises d'État chinoises émargent au budget de l'État; pour reprendre une expression chinoise : elles «mangent à la grande marmite commune ». C'est le budget de l'État qui finance les besoins de trésorerie et les investissements, absorbe les profits et éponge les pertes. Les entreprises sont finalement un simple maillon de l'économie nationale et du budget de l'État, leur comptabilité relève du domaine de la comptabilité publique ${ }^{3}$.

Leur système comptable est basé sur la notion de fonds. Le document

\footnotetext{
${ }^{3}$ D'ailleurs, jusqu'au milieu des années 80 , la comptabilité n'existe pas en tant que discipline indépendante, elle est simplement considérée comme une spécialité des finances publiques.
}

Corine Eyraud. Sociologie du Travail, 2003, n 4, pp. 491-508. 
essentiel est la «balance de fonds», il se compose de deux parties équivalentes (les fonds alloués et les fonds utilisés), chacune divisée en trois catégories : les fonds fixes, les fonds circulants et les fonds spéciaux. La gestion financière des entreprises doit obéir au principe «d'utilisation des crédits conformément à leur affectation ». Théoriquement, le jeu de documents comptables comprend un «tableau des profits », mais de nombreuses entreprises ne le remplissent pas, ce qui montre clairement que la question du profit est d'ordre secondaire.

Les définitions de nombreuses catégories comptables sont directement liées aux concepts de l'économie politique marxiste. Les fonds fixes, par exemple, sont définis comme «les fonds utilisés par l'entreprise en tant que moyens de travail »; ils comprennent les bâtiments professionnels, les équipements, les machines mais aussi tout «ce qui rend possible pour les travailleurs la réalisation de leur travail sur une longue période » (logements, crèches et écoles pour les enfants, dispensaire, etc.). Il s'agit clairement d'une tout autre idée et d'une tout autre forme d'entreprise que celle que nous connaissons aujourd'hui en Occident : c'est l'entreprise qui assume les coûts de production de la force de travail, ces coûts sont intégrés aux coûts de production stricto sensu.

Dans la théorie marxiste, le profit est la valeur ajoutée par les travailleurs. Les impôts sont donc considérés comme du profit transféré à l'État, les frais financiers comme du profit transféré aux banques. Dans le système comptable de l'économie planifiée et ce jusqu'à la réforme de 1993, le bénéfice ou profit comprend donc les impôts et taxes diverses, les frais financiers (ils sont rares mais

Corine Eyraud. Sociologie du Travail, 2003, n 4, pp. 491-508. 
existent), le profit distribué aux employés par le fonds de bien-être (dédié à la construction de logements, crèches, écoles, etc.) et par les primes, et le profit remis à l'État. Les impôts, les frais financiers, les primes des employés (mais non pas les salaires) ne sont donc pas considérés comme des charges mais comme des répartitions du bénéfice (voir Eyraud, 1999, p.268, et de manière beaucoup plus générale Mangenot, $1976^{4}$ ).

\subsection{Quelle comptabilité pour quel type de gestion de l'économie?}

La comptabilité des entreprises est non seulement basée sur la pensée économique marxiste mais se fonde et s'intègre dans le système économique particulier qu'est celui de l'économie planifiée. Ses objectifs sont, d'un point de vue macro-économique fournir des informations pour la planification nationale, d'un point de vue micro-économique contrôler la réalisation du plan par l'entreprise et l'application de la discipline financière édictée par l'État et les autorités locales.

Étant donné cet objectif macro-économique, le lien entre système comptable et système statistique est très fort : ce sont, le plus souvent, les catégories statistiques qui informent les catégories comptables. Ainsi, le système

\footnotetext{
${ }^{4}$ Cela pourrait servir de base à des débats intéressants : il n'est effectivement pas neutre de concevoir une opération économique comme une charge, un investissement ou une répartition du bénéfice, et la décision est discutable. Plus près de nous, et dans le temps et dans l'espace, le Centre des Jeunes Dirigeants d'entreprise a proposé d'inverser l'équation de base de l'entreprise, en considérant que la rémunération du capital devrait être comptabilisée comme une charge, et que la différence entre les produits et les charges - le bénéfice - devrait profiter uniquement aux salariés : «c'est un moyen d'attribuer les résultats aux collaborateurs de l'entreprise qui en sont la finalité, et finalement de remettre la finance au service de l'économie » (CJD, 1996, p.106). Cette idée fut mise en pratique sous différentes formes par des patrons socialistes et plus précisément fouriériste au $19^{\mathrm{e}}$ siècle (par exemple par J.B. Godin dans son Familistère de Guise) puis par des patrons catholiques sociaux au $20^{\mathrm{e}}$ siècle (par exemple A. Dubois dans les Forges et Aciéries de Bonpertuis).
}

Corine Eyraud. Sociologie du Travail, 2003, n 4, pp. 491-508. 
statistique comprend une donnée intitulée «le montant global des marchandises au détail dans la société ». Pour obtenir cette donnée, il est demandé aux entreprises industrielles et commerciales de fournir dans leurs documents comptables non seulement leur montant des ventes (en valeur et en volume) mais également le «montant net des ventes » (en valeur et en volume) qui ne comprend que les ventes achetées par les clients pour être consommées. « Le montant global des marchandises au détail dans la société » est le total des «montants nets des ventes »de toutes les entreprises nationales; cette donnée est utilisée premièrement pour décider de l'augmentation ou de la diminution de telle production d'un point de vue national ou local, voire de transferts de tel produit entre provinces ou municipalités, deuxièmement pour vérifier l'adéquation entre la destination prévue par le plan et la destination effective. Cette subordination du système comptable au système statistique se retrouve également dans la comptabilité bancaire : les documents comptables des banques sont ainsi élaborés secteur industriel par secteur industriel, dans un même souci d'information pour la planification et de vérification.

L'objectif micro-économique de contrôle sur l'entreprise se réalise essentiellement par l'application du principe «d'utilisation des crédits conformément à leur affectation »; contrôle renforcé par le système de relations banque/entreprises. Toute entreprise chinoise doit obligatoirement ouvrir ses comptes en banque dans le bureau de banque de son district. Elle détient un compte bancaire pour chaque type de fonds, soit un pour les fonds fixes, un pour les fonds circulants, un pour les fonds spéciaux. Elle doit fournir à sa banque son

Corine Eyraud. Sociologie du Travail, 2003, n 4, pp. 491-508. 
plan d'achat, son plan de vente, son plan de coût et son plan financier, ainsi que ses documents comptables mensuels, trimestriels et annuels. Sur le plan d'achat figurent les matériaux à acheter, leurs prix et l'identité des fournisseurs, sur le plan de vente les produits à vendre, leurs prix et l'identité des clients, sur le plan financier l'utilisation prévue de chacun des fonds. Jusqu'au début des années 1990, il n'existe en Chine qu'un seul moyen de paiement ${ }^{5}$ : charger la banque, en lui fournissant la facture, d'aller se faire payer auprès de la banque du client ou d'aller payer la banque du fournisseur, il est donc facile pour la banque de vérifier que l'entreprise se conforme bel et bien au plan. Finalement, la banque chinoise non seulement contrôle financièrement les entreprises, mais assure quasiment leur gestion financière.

\subsection{Les pratiques économiques et comptables des entreprises}

Une très large majorité des activités économiques des entreprises se déroulent donc au sein du plan ${ }^{6}$, mais l'économie hors-plan existe également même s'il est difficile d'évaluer sa place. Les entreprises d'État semblent y avoir recours principalement pour pallier les ruptures des approvisionnements inscrits dans le plan, ou pour obtenir des produits rares difficiles à obtenir par la voie officielle. Voici ce que nous en dit un de nos interlocuteurs : «Quand j'étais responsable d'atelier, une de nos machines s'est cassée, nous n'arrivions pas à acheter la pièce de rechange principalement produite à Shanghai et à Shihan. A

\footnotetext{
${ }^{5}$ Les autres moyens de paiement, tels les chèques ou les effets de commerce, sont considérés comme des « outils capitalistes » et ont été abolis au début des années 1950.

${ }^{6}$ Cette question se complexifie quand on sait que les différents plans qui encadrent les activités des entreprises ne proviennent pas toujours du même organisme administratif, des problèmes d'incohérence voire d'incompatibilité se posent parfois.
}

Corine Eyraud. Sociologie du Travail, 2003, n 4, pp. 491-508. 
cette époque l'offre ne satisfaisait pas la demande, c'était très bon marché mais tu n'arrivais pas à te procurer la pièce, il aurait fallu leur permettre d'être un peu plus cher, et avec cet argent de se développer, mais à cette époque sans l'autorisation des organismes compétents ils ne pouvaient pas mener à bien ce développement, alors il valait mieux que ce soit d'autres qui le fassent. C'est une entreprise gérée par une commune populaire qui l'a fait, ce qu'elle produisait n'était pas bon du tout, en plus c'était très cher, mais nous sommes tout de même aller lui acheter cette pièce ».

Ces activités en-dehors du plan peuvent prendre différentes formes: l'achat direct à l'entreprise productrice comme ci-dessus, le troc entre organisations industrielles - troc souvent différé -, mais aussi l'achat dans des centres d'approvisionnements non officiels. Ces activités semblent considérées à l'époque non pas comme des activités illégales relevant de la délinquance et passibles de sanctions disciplinaires, mais comme des activités simplement non officielles, s'inscrivant dans la part d'autonomie laissée à l'entreprise prise en tant qu'acteur, et aidant la planification et l'allocation des ressources.

Il est toutefois difficile de savoir comment ces opérations hors plan figurent dans la comptabilité de l'entreprise, et comment les paiements qu'elles induisent parfois sont négociés avec la banque et l'autorité de tutelle. De manière générale, le problème de la «falsification» des comptabilités et statistiques communistes afin de les mettre en conformité avec le plan a été soulevé par de très nombreux analystes. Mais ce décalage entre comptabilité normalisée et comptabilité pratiquée est, nous semble-t-il, une constante dans l'histoire

Corine Eyraud. Sociologie du Travail, 2003, n 4, pp. 491-508. 
comptable; ce qui change ce sont les objectifs avec lesquels on la met en conformité ou autrement dit les formes de pouvoir avec lesquelles ces objectifs sont en relation : dans une comptabilité à objectif fiscal comme c'est le cas en France, différentes techniques comptables sont utilisées afin de diminuer le résultat et donc le montant de l'impôt sur les sociétés, le résultat n'a alors pas grand «sens économique »; la faillite d'Enron a montré récemment un autre type de décalage : «Enron cristallise l'ensemble des failles des pratiques comptables qui permettent de répondre aux impératifs de rentabilité provenant du nouveau pouvoir des actionnaires. (...) Les normes de rentabilité exigées par les marchés financiers étaient telles qu'elles ont amené certains dirigeants à afficher des bénéfices artificiels et ainsi garder leur confiance » (Ferrandon, p.69) par exemple en externalisant une partie de leurs pertes dans des structures financières appelées special purpose entities (SPE) non consolidées au bilan de l'entreprise.

Analyser la comptabilité réellement pratiquée par les entreprises nécessite, en Chine ou ailleurs, d'entrer très précisément dans les comptes et dans la manière dont ils sont constitués. L'accès à un tel matériau est très difficile, voire quasi impossible, c'est pourquoi nous avons centré notre analyse sur la comptabilité normalisée ou cadres comptables.

\section{4. Élaboration des cadres comptables et mode de gestion du social}

Il existe, à cette époque, plus d'une cinquantaine de «systèmes comptables » et de «systèmes financiers ». Les premiers comprennent un plan comptable et un modèle type de chaque document comptable, les seconds sont des textes très détaillés et très directifs expliquant le contenu de chaque item

Corine Eyraud. Sociologie du Travail, 2003, n 4, pp. 491-508. 
comptable, sa méthode de comptabilisation, etc. Ces systèmes comptables et financiers diffèrent selon le type d'entreprise (entreprises collectives ou entreprises d'État) et selon le secteur de production, puisque chaque ministère industriel les élabore, à partir des directives du ministère des Finances et du bureau national des Statistiques, pour son propre secteur. Ces systèmes ont valeur de règlements; la division comptabilité du ministère industriel, mettons $\mathrm{du}$ ministère de la Sidérurgie, les transmet aux services comptabilité des bureaux provinciaux de la sidérurgie qui les transmettent aux services comptabilité des bureaux municipaux de la sidérurgie, qui les transmettent finalement aux sections comptabilité des entreprises qui leur sont subordonnées.

L'information comptable effectue le chemin inverse: les documents mensuels, trimestriels et annuels sont remis par l'entreprise à son autorité de tutelle, par exemple le bureau municipal de la sidérurgie, à sa banque et au bureau des Finances. Il est quasiment impossible pour tout autre organisme (entreprise ou administration) d'obtenir ces documents ${ }^{7}$. L'autorité de tutelle évalue, grâce à ces documents, le degré de réalisation du plan de toutes les entreprises qui lui sont subordonnées, et remet ces données agrégées au bureau local des Statistiques et au bureau provincial de la sidérurgie. Ce dernier agrège les données de toute la province puis les remet au bureau provincial des Statistiques et au ministère de la Sidérurgie.

On retrouve dans ce mode de gestion de l'information et des systèmes comptables et financiers, les principaux éléments du système chinois de gestion de

\footnotetext{
${ }^{7}$ Ceci dit, dans ce système économique-là, cela n'a que peu d'intérêt et peu de sens.
}

Corine Eyraud. Sociologie du Travail, 2003, n 4, pp. 491-508. 
l'économie et, plus largement, de gestion de la société, que nous avons développés par ailleurs (Eyraud, 1999) :

* le système de «gestion échelon par échelon » des unités chinoises : il existe très peu de lois, tout est encadré par des règlements transmis en suivant les lignes administratives qui relient hiérarchiquement les organisations chinoises, et pouvant être modifiés (en raison de conditions sectorielles ou locales particulières) par un ministère pour son secteur industriel ou par les échelons provinciaux et municipaux ; cela signifie qu'il s'agit d'un type de gestion très particulariste et non pas universaliste fondé sur un droit national. Les entreprises reçoivent par ce système de «gestion échelon par échelon» tous les règlements qui encadrent leurs activités, par exemple tout ce qui concerne l'embauche, les salaires, les conditions de travail, d'hygiène, etc.

* une gestion très précise, très détaillée, qui réduit très largement les possibilités de choix au niveau de l'organisation (ici au niveau de l'entreprise et en matière d'enregistrement comptable) ;

* le cloisonnement de l'information (économique) au sein de chaque grand secteur industriel, seuls les bureaux des statistiques transcendent localement ces cloisonnements verticaux.

\section{Encadré n ${ }^{\circ} 1$ : L’Usine de Médicaments de Kunming}

Le personnel de l'usine est composé de 1000 employés en activité et 400 retraités. Une fois affecté dans l'entreprise, on est employé à vie, on peut même, à la retraite, recommander son successeur (en général son enfant), ce système d'héritage de l'emploi est très courant jusqu'au milieu des années 80 .

Corine Eyraud. Sociologie du Travail, 2003, n 4, pp. 491-508. 
L'usine fournit un logement à tout son personnel. Les employés (en activité ou retraités) prennent leur repas à la cantine le midi et parfois le soir, et obtiennent gratuitement une partie des aliments de base au magasin de l'entreprise. Ils disposent également d'une crèche, d'écoles (de la maternelle au lycée), d'un hôpital (avec 30 médecins), mais aussi d'un commissariat chargé de la sécurité sur l'espace (clos) de l'usine et des activités d'état civil : gestion des permis de résidence, des formalités de mariage, naissance, etc. Les personnels de ces structures sont rétribués et logés par l'entreprise.

L'autorité de tutelle de l'usine est un organisme de gestion du secteur pharmaceutique, selon les périodes, de niveau central, provincial ou municipal ; depuis 1970 il s'agit du Bureau de Gestion des Médicaments de la province du Yunnan. Il remet à l'usine les différents plans qui encadrent ses activités économiques, et les règlements applicables en matière de gestion financière, gestion du personnel, sécurité, etc. ; cette dernière lui remet toutes les informations concernant ses activités. On trouve au sein de l'usine une organisation du Parti (être membre du Parti c'est être membre du comité du Parti de son usine), et une organisation syndicale. Chacun des éléments de l'usine (organisation, département...) est subordonné à son homologue au sein de l'autorité de tutelle, lui-même étant l'aboutissement d'organismes correspondant au niveau local et national. Le commissariat, quant à lui, dépend du district, tout comme la structure de planification des naissances, dans les périodes où elle existe.

\section{La réforme comptable et ses enjeux}

A partir du début des années 1980, le monde académique chinois commence à avoir accès aux théories et aux systèmes comptables occidentaux, en particulier anglo-saxons. L'idée de réformer le système chinois commence à poindre.

Corine Eyraud. Sociologie du Travail, 2003, n 4, pp. 491-508. 
Différents groupes d'experts et commissions sont créés tout au long des années 1980, des colloques internationaux ${ }^{8}$ sont organisés au début des années 1990. Et en 1993, de nouveaux règlements organisant cette réforme comptable entrent en vigueur $^{9}$; l'idée générale est explicite: il s’agit de «se connecter avec les pratiques internationales ». Certains acteurs internationaux vont d'ailleurs jouer un rôle important et favoriser cette connexion : la Banque mondiale va accorder un prêt à la Chine pour ce projet de réforme à condition qu'elle accepte un cabinet d'expertise comptable international en tant que conseiller sur cette réforme auprès du ministère des Finances, ce sera un des cinq grands cabinets anglo-saxons.

\subsection{Changement d'objectifs et détachement d'avec la statistique}

Ces nouveaux ${ }^{10}$ règlements introduisent tout d'abord un changement de portée générale: l'information comptable doit être élaborée dans le but de « répondre aux besoins du contrôle macro-économique, des utilisateurs extérieurs et de la gestion financière des entreprises ». Les états financiers deviennent des documents externes, ouverts aux utilisateurs extérieurs et non plus aux seuls

\footnotetext{
${ }^{8}$ Nous avons participé à deux de ces colloques organisés par le Ministère des finances chinois, l'un en 1993 en tant qu'observatrice, le second en 1994 en tant qu'interprète (une délégation française était présente) ; le premier était destiné à faire le point sur la réforme comptable de 1993, le second à en étudier les «nécessaires » développements.

${ }^{9}$ Là aussi nous avons travaillé essentiellement sur des sources en langue chinoise, mais une explicitation plus précise de cette réforme peut se trouver dans Tang, 1994, et en langue française dans Pairault (dir.), 2000, Le plan comptable chinois, Editions de l'ordre des experts comptables, Paris.

${ }^{10}$ Le terme «nouveau » est utilisé en rapport avec le système comptable précédent, mais il ne doit pas nous faire oublier les « lignes de continuité » qui peuvent exister avec la tradition comptable chinoise (cf. Goody, 1999, pp. 100-105, ainsi que Shanghai University..., 1987, pp. 165-190). C'est de fait le système de l'économie planifiée qui est en rupture quasi-totale avec la tradition comptable impériale et républicaine. Il est d'ailleurs intéressant de noter que dans les années 80 , en même temps que se créent groupes d'experts et commissions autour de la réforme comptable, se développent les recherches portant sur l'histoire de la comptabilité chinoise «d'avant la Révolution ».
}

Corine Eyraud. Sociologie du Travail, 2003, n 4, pp. 491-508. 
bureaux administratifs concernés; les documents de comptabilité analytique deviennent, en revanche, des documents internes que l'entreprise ne remet plus obligatoirement aux départements administratifs et que le directeur établit à sa convenance. La comptabilité change donc officiellement d'objectif et de destinataires: il ne s'agit plus seulement de fournir des informations pour la planification à venir et pour le contrôle de la planification en cours, mais de fournir des informations «économiques » sur l'entreprise, pouvant être utilisées comme un outil de gestion par la direction et permettant une évaluation de l'entreprise par ses différents partenaires.

L'information comptable va donc se détacher de l'information statistique. Nous pouvons analyser ce phénomène dans le processus d'élaboration des systèmes comptables (plan comptable et modèles standards des documents comptables). Pendant l'économie planifiée, ces systèmes étaient élaborés par les ministères industriels sous la direction du bureau national des Statistiques et du ministère des Finances. Dans les années 1980, ils sont élaborés dans une négociation de plus en plus difficile entre ces différents acteurs, et en particulier entre le ministère industriel représentant les intérêts des entreprises et promouvant une comptabilité ancrée dans la logique économique et utile à l'entreprise, et le bureau national des Statistiques promouvant quant à lui une comptabilité orientée vers la planification et les statistiques nationales. La logique entrepreneuriale finit par l'emporter : à partir de 1993, les documents comptables et les documents statistiques sont dissociés et le bureau national des Statistiques n'intervient plus dans l'élaboration des systèmes comptables.

Corine Eyraud. Sociologie du Travail, 2003, n 4, pp. 491-508. 
Le même phénomène de détachement de l'information comptable de l'information statistique se retrouve dans la comptabilité bancaire, elle aussi réformée en 1993 et 1994. Un de nos interlocuteurs, travaillant dans une banque, nous explique cette réforme : «Avant 1992, nous subissions l'influence de la planification, les items de notre balance de fonds étaient tous divisés en fonction du secteur: chimie, textile, etc. Pour nous banques, cela n'avait aucune utilité, pour le bureau national des Statistiques oui, mais nous ce qui nous intéresse c'est de savoir par exemple s'il s'agit de comptes à vue ou de comptes à échéance fixe, maintenant nous possédons ces informations. »

\subsection{Nouvelles catégories comptables, nouvelle forme d'entreprise et nouveau mode de gestion du social ${ }^{11}$ ?}

Les règlements promulgués en 1993 définissent les nouveaux concepts utilisés en comptabilité : l'actif, le passif, les capitaux propres, un produit, une charge, un profit et une perte. Ils définissent également les nouveaux documents comptables $^{12}$, qui seront désormais un bilan, un compte de résultat et un tableau de financement. Ce nouveau système comptable, avec la notion de bilan, de capitaux propres et la séparation qu'il instaure entre la comptabilité réalisée pour l'extérieur et la comptabilité interne ou comptabilité analytique, se fonde sur un certain modèle de l'entreprise : une entreprise (ou une banque) est une entité à part entière, une entité patrimoniale, elle n'est plus un simple élément du système

\footnotetext{
11 «Social» est ici pris à double sens : nous parlerons à la fois du mode de gestion de la société (comme nous l'avons fait dans la première partie de cet article), et à la fois du mode de gestion des activités sociales (« opposées » aux activités économiques).

12 Ces documents sont élaborés sur le modèle anglo-saxon, légèrement différent du modèle français ou du modèle allemand. En fait, l'influence de la comptabilité anglo-saxonne est considérable.
}

Corine Eyraud. Sociologie du Travail, 2003, n 4, pp. 491-508. 
économique national et du budget de l'État. La comptabilité des entreprises chinoises devient ainsi une comptabilité privée, une comptabilité d'entreprise au sens capitaliste du terme : elle vise à évaluer un patrimoine et à calculer un résultat $^{13}$. Si l'on ajoute à cela le fait que les immobilisations non professionnelles (logements des employés, crèches, etc.) ne doivent plus, à terme, figurer dans le bilan des entreprises, on voit se dessiner plus précisément le modèle de l'entreprise qui fonde cette réforme : une entité essentiellement économique qui ne doit plus assumer ni les coûts de production de la force de travail ni la gestion de cette production, mais qui externalise et ces coûts et cette gestion.

Mais qui va alors les assumer? La Chine a opté pour une solution mixte : une socialisation de certains de ces coûts - socialisation qui se fait non plus au niveau de l'entreprise mais au niveau des municipalités, des provinces et/ou de l'État avec la mise en place d'un système d'assurances maladie (jusque là assumées directement par l'entreprise) et chômage (les surplus de main d'œuvre étaient gérés par l'entreprise) -, et une individualisation des coûts de formation (école publique, obligatoire et payante) et du système de retraite (retraite essentiellement par capitalisation). De manière générale, on assiste aujourd'hui en Chine à ce que Jacques Donzelot (1984) a appelé «l'invention du social », c'està-dire à la mise en place d'une administration du social autonome et spécialisée, puisque ces activités et cette administration existaient bel et bien dans l'entreprise

${ }^{13}$ Il devient d'autre part possible pour les entreprises de réaliser des provisions pour créances douteuses ; est donc reconnu ce que l'on appelle en comptabilité le principe de prudence. Pendant l'économie planifiée, c'est l'État qui était, au bout du compte, responsable de tout achat, vente, perte ou profit, et l'on ne pouvait pas douter de sa solvabilité. L'activité économique devient en Chine une activité à risques. Finalement, la réforme comptable supprime le principe "d'utilisation des crédits conformément à leur affectation", les directeurs d'entreprise sont ainsi censés acquérir une plus grande liberté quant à l'utilisation de leurs capitaux.

Corine Eyraud. Sociologie du Travail, 2003, n 4, pp. 491-508. 
d'État mais étaient « encastrées » dans les activités du personnel dirigeant.

Ainsi là où il y avait auparavant un seul système économique national et où les différentes dimensions de l'activité de production étaient totalisées dans l'entreprise, les différentes dimensions de l'activité économique se trouvent aujourd'hui progressivement séparées en différents domaines spécialisés gérés par des organisations elles-mêmes spécialisées. Des frontières se créent, des découpages, des discontinuités entre une entreprise d'État et les autres entreprises d'État (chacune ayant son bilan et ses capitaux propres), entre les entreprises et les départements administratifs, entre les entreprises et les banques d'État, entre les entreprises et l'administration du social et donc entre ce que nous appelons activités économiques et activités sociales, entre le travail et le non-travail (chômage et retraite). Ces découpages, ces séparations définissent ce que Robert Salais (1986) a appelé les internalités et les externalités ${ }^{14}$ de l'entreprise ; et elles sont inscrites, objectivées dans les nouveaux documents, catégories et règles comptables.

Les nouveaux règlements comptables, d'autre part, sont applicables à toutes

${ }^{14}$ Il y aurait beaucoup à dire sur cette question des externalités (Callon, 1999 ; Friedberg et Musselin, 1999). Si l'on s'en tient à l'objectivation de ces externalités dans la comptabilité, on peut signaler les travaux d'un groupe de chercheurs, composé en particulier de comptables, visant l'élaboration d'un «bilan sociétal»: «L'entreprise peut enrichir ou au contraire appauvrir son milieu par des coûts externés plus ou moins maitrisés, non seulement en matière d'approvisionnement, de pollution, mais aussi en capital humain. (...) Ce qui est désormais nouveau, c'est le besoin d'évaluer les actions réalisées pour assumer cette responsabilité sociale ou à l'inverse les externalités négatives que fait subir l'entreprise à son environnement. (...) Cela ne peut se faire qu'en changeant les grilles de lecture à l'aune desquelles nous jugeons la bonne santé de l'entreprise, et cela implique d'avoir une autre conception de la performance. (...) L'idée de bilan sociétal est née dans ce contexte avec pour objectif d'identifier les éléments de production et d'échanges de l'entreprise envers la société. » (Capron, Leseul, 1997, p.30) Ce bilan est élaboré à partir d'un questionnaire qui comprend près de 450 items décomposés en 9 domaines d'investigation (Activité et relations clients; Gestion économique; Anticipation, innovation, prospective; Production, organisation du travail; Ressources humaines; Acteurs internes, citoyens de l'entreprise; Environnement humain, social et institutionnel; Environnement biophysique ; Finalités, valeurs, éthique).

Corine Eyraud. Sociologie du Travail, 2003, n 4, pp. 491-508. 
les entreprises installées sur le sol chinois, quels que soient leur statut et leur secteur de production. Les systèmes comptables et financiers devraient disparaître pour être remplacés par un seul plan comptable, lui aussi applicable à tous. En cela, cette réforme s'inscrit dans un changement fondamental de mode de gestion de la société : le passage d'une micro-gestion particulariste de l'échelon inférieur par l'échelon supérieur à une gestion universaliste par la loi et par des organismes spécialisés dans une activité donnée (ici le ministère des Finances et les organismes professionnels comptables). Ce changement est en cours dans la société chinoise ; dans de nombreux domaines d'activités, le gouvernement fait un effort important de codification et crée des organismes spécialisés en charge d'élaborer ce droit, de protéger ces droits, de diffuser et conserver l'information.

\section{Encadré nº : La SA de Médicaments de Kunming}

Des réformes sont dès 1979 expérimentées dans des entreprises pilotes, l’Usine de Médicaments de Kunming en est une. On lui permet, dès 1981, de conserver une partie de son bénéfice, elle va ainsi pouvoir se moderniser et s'agrandir. Le IIIe plenum du Comité central décide en 1984 la mise en place d'une «économie de marchandises planifiée ». L'usine va ainsi se mettre à vendre une partie de ses produits, à décider elle-même de sa production au-delà du plan et du prix de ses nouveaux produits. Le réseau commercial chinois se diversifie rapidement. Un «contrat de responsabilité » est signé entre le gouvernement et le directeur. En tant qu'entreprise pilote, l'usine signe un contrat très avantageux, la part des bénéfices qui lui sont laissés augmente fortement ; très vite elle ne reçoit plus de plan de production, et très peu d'instructions de son autorité de tutelle, le plus souvent elle traite directement avec le gouvernement provincial. Elle expérimente le

Corine Eyraud. Sociologie du Travail, 2003, n 4, pp. 491-508. 
système d'emploi contractuel : les nouveaux employés sont recrutés par l'usine dans la limite des quotas d'embauche qui lui sont alloués, et embauchés sur contrat à durée déterminée; les salaires et les primes deviennent liés au bénéfice de l'entreprise et au rendement de l'atelier. Finalement, les prêts bancaires remplacent progressivement les allocations budgétaires; en tant qu'entreprise pilote, l'usine a un accès privilégié aux prêts bancaires. Le système bancaire chinois commence à se diversifier, ce processus s'accélèrera largement dans les années 90 .

En 1992, suite au voyage bien connu de Deng Xiaoping dans le sud de la Chine, les réformes vont connaitre un nouvel essor, le XIVe congrès décide la mise en place d'une «économie socialiste de marché ». Il s'agit de transformer les entreprises d'Etat en sociétés par actions, d'externaliser leurs fonctions sociales, de développer un marché du travail et un système d'assurances sociales, d'encourager le développement du secteur privé et du secteur tertiaire, de créer un marché du capital et d'approfondit la réforme du système bancaire, de mettre en place un système fiscal, de libéraliser le commerce extérieur, et de développer un droit commercial. La réforme comptable est mise en place en 1993 ; la loi des Sociétés, très proche de ce qui se pratique en droit international, entre en vigueur en 1994, tout comme un nouveau système fiscal (avec TVA...) et une quasiconvertibilité du yuan.

En 1995, la S.A. de médicaments de Kunming est créée, son capital est composé des immobilisations professionnelles de l'usine (35\% du capital), et de l'apport de trois autres entreprises d'État et du personnel (dont les parts représentent plus de $20 \%$ du total). L'autorité de tutelle de l'usine ne participe pas à l'assemblée des actionnaires. L'usine existe toujours, mais ne produit plus, elle gère les activités sociales (logements, retraites, hôpital, écoles...) et les assume grâce aux dividendes qu'elle touche de la S.A. ; le PDG de la S.A. reste directeur de l'usine. Tout le personnel est à présent embauché sur 
contrat. La S.A. est gérée essentiellement dans une logique d'économie de marché ; elle crée une entreprise à capitaux mixtes avec une société américaine en 1997.

La S.A. ne sera pas concernée par l'accélération des réformes qui suivra, en 1997, le discours de Jiang Zemin au XVe Congrès et l'accession de Zhu Rongji au poste de premier ministre (licenciements, mises en faillite) ; son statut d'entreprise pilote lui a permis en 15 ans de devenir une entreprise rentable. L'usine, quant à elle, continue de se défaire progressivement de ses activités sociales. Ainsi après une augmentation continue des loyers, les logements ont été vendus aux employés ; l'hôpital a fermé ; la crèche et l'école maternelle continuent de fonctionner mais sont devenues payantes; les enfants à partir de 6 ans vont dans les écoles du district qui ont du se développer et qui sont elles aussi devenues payantes; finalement depuis 1998 l'entreprise ne paye plus les retraites mais payent des cotisations sociales.

Dans les faits, la relation de subordination de la S.A à l'autorité de tutelle de l'usine perdure: le type de «gestion échelon par échelon» reste, malgré des changements importants, la méthode de circulation et d'application de nombreux règlements. En outre, le comité du Parti et le syndicat sont communs à la S.A. et à l'usine; en l'absence de réformes politiques ne serait-ce qu'organisationnelles, ils continuent à être subordonnées aux organisations équivalentes de l'autorité de tutelle. Le PDG de la S.A. rend donc des comptes au conseil d'administration, mais aussi en tant que membre du Parti et directeur de l'usine au comité du Parti de l'autorité de tutelle, il peut ainsi se trouver confronté à des conflits d'intérêts et de logiques. C'est le cas en 1999 : le directeur de l'autorité de tutelle en désaccord avec l'orientation des réformes promues par le gouvernement provincial et dont la S.A. est emblématique décide d'en destituer le PDG. Certains organismes provinciaux répondent violemment, le cas est présenté à Jiang Zemin, alors président de la République, qui se déclare très satisfait de l'évolution de cette entreprise, 
le PDG est réintégré, le directeur de l'autorité de tutelle est limogé, mais l'entreprise a connu plusieurs mois de flottements et mettra plusieurs mois à s'en remettre.

C'est évidemment un processus simplifié que nous venons de présenter, les réformes ont été marquées par des coups d'arrêt, des accélérations, des différences régionales et des disparités entre entreprises.

Il est possible de réfléchir à ce lien entre formes comptables, forme d'entreprise et mode de gestion du social (tel qu'entendu ici) en prenant appui sur différents travaux d'économie ou de sociologie. Les économistes néoinstitutionnalistes ont par exemple montré que la transition d'une économie planifiée à une économie de marché supposait la destruction d'institutions ${ }^{15}$ anciennes et la construction de nouvelles (Vercueil, 2001); ils ont ainsi mis l'accent sur l'existence de « configurations institutionnelles », et sur le fait que ce processus de destruction et création d'institutions est « dépendant du chemin déjà parcouru » («path dependancy », North, 1990).

L'école de la régulation a elle aussi développé la notion de «configuration institutionnelle » et largement montré la complémentarité des arrangements ou compromis institutionnels contribuant à la viabilité d'un mode de régulation. Robert Boyer analysant la transition des économies de l'Est (1993) a parlé de «grande transformation », estimant qu'elle nécessiterait au minimum une génération, soit deux à trois décennies.

De nombreux travaux de l'école des conventions ont également insisté sur la

15 Pour une analyse de la notion d'institution chez les économistes institutionnalistes, on peut se référer à Vercueil, 2001, pp. 268-277.

Corine Eyraud. Sociologie du Travail, 2003, n 4, pp. 491-508. 
cohérence et la continuité de formes conventionnelles très diverses, par exemple dans un même modèle de production industrielle (voir l'analyse du fordisme par Laurent Thévenot, 1985).

De manière générale, il faut bien dire que la mise en relation d'institutions différentes, que le questionnement sur leur coprésence, sur l'aspect nécessaire ou pas de leurs relations, ainsi que la mise en rapport de ces institutions avec un tout social plus ou moins large, est une des caractéristiques des sciences sociales, et en particulier de la sociologie.

En ce qui nous concerne, nous avions choisi dans nos travaux précédents (Eyraud, 1999) de concevoir l'entreprise d'Etat chinoise comme une forme sociale particulière, et de centrer notre analyse sur deux de ses aspects : ses contours et sa qualité essentielle. L'entreprise d'Etat du temps de l'économie planifiée est ainsi d'une part un ensemble relativement flou qui se prolonge de maintes manières dans l'organisation administrative, politique et sociale, d'autre part plus qu'un lieu de travail, plus qu'une communauté de vie, une institution sociale totale, au sens d'Erving Goffman. Le nouveau type d'entreprise que les réformes visent à mettre en place a, en revanche, des contours bien délimités, ces entreprises devenant des entités à part entière, essentiellement économiques (voir encadrés $n^{\circ} 1$ et 2). Ces deux types de formes sociales sont objectivées dans des documents comptables particuliers, on peut ainsi « lire » ce changement dans la réforme comptable. Mais très vite, ces interventions sur l'entreprise ont impliqué, ont nécessité une intervention sur la société dans son ensemble; ainsi les réformes touchant à la société globale sont, en grande partie, le produit des réformes visant l'entreprise.

Corine Eyraud. Sociologie du Travail, 2003, n 4, pp. 491-508. 
Une telle recherche sur l'entreprise d'État chinoise et ses réformes - dont la réforme comptable est emblématique - apporte en retour des connaissances sur les fondements sociétaux de l'entreprise occidentale contemporaine : l'existence de l'entreprise occidentale, sous la forme que nous lui connaissons, est ainsi inséparable de la structuration particulière de notre espace social, d'un mode particulier de gestion de la société, et de l'existence d'un grand nombre d'organisations. Nous pouvons avancer que la réunion de ces différents éléments est une condition de possibilité de l'existence de ce type d'entreprise et de son mode particulier de fonctionnement et de relations à son environnement. C'est très certainement du fait de cette congruence nécessaire et de la coexistence aujourd'hui en Chine d'éléments très hétérogènes que la réforme comptable n'a pour l'instant qu'une efficacité limitée.

\subsection{L'efficacité sociale de la réforme comptable}

Il semble admis aujourd'hui - après les travaux des ethnométhodologues sur le rôle que jouent les textes et documents dans la construction sociale de la réalité et après les travaux sur les catégorisations sociales ${ }^{16}$-, que ces formes sociales objectivées, puisqu'elles véhiculent des modèles de représentation de la réalité, ont un effet « en retour » sur la réalité sociale : elles coordonnent et structurent les activités et les anticipations des acteurs. On peut ainsi s'attendre à ce que le modèle rationnel qui fonde les nouveaux documents et catégories comptables informe, en partie, les pratiques non seulement des comptables mais également

${ }^{16}$ L. Boltanski (1982), A. Desrosières et L. Thévenot (1988), ou R. Salais (1986) pour ne citer qu'eux.

Corine Eyraud. Sociologie du Travail, 2003, n 4, pp. 491-508. 
des directeurs d'entreprises et des autres acteurs économiques ; en cela la nouvelle comptabilité pourrait avoir une certaine efficacité sociale et être un des moteurs du changement social en cours aujourd'hui dans la société chinoise.

Nous allons donc tenter d'analyser, non pas les changements que ces nouveaux cadres comptables (ou comptabilité normalisée) induisent dans la comptabilité pratiquée (nous l'avons dit nous n'avons pas les matériaux nécessaires), mais l'action de ces cadres comptables premièrement sur les représentations - et ce à travers l'émergence de nouvelles catégories économicocomptables chez les acteurs eux-mêmes -, deuxièmement sur les pratiques - à travers l'utilisation des bilans comptables à la fois dans les décisions bancaires d'octroi ou refus de prêts et dans les décisions gouvernementales de mises en faillite. Pour ce faire nous allons comparer les entretiens et informations recueillis lors de nos enquêtes de terrain du milieu des années 1990 avec ceux recueillis à la fin des années 1990 et début des années 2000. Les moments de ces observations restent très proches étant donnée l'ampleur des transformations dans lesquelles prend place cette réforme comptable, mais des changements sont, comme nous allons le voir, déjà perceptibles.

\subsubsection{Milieu des années 1990}

Prenons, pour commencer, l'exemple des notions de prêt, d'investissement, de subvention, de dettes et de créances; toutes sont des nouvelles catégories définies par la réforme comptable. Trois ans après la mise en place de cette réforme, elles sont encore largement confondues dans les discours des directeurs d'entreprise et du personnel bancaire : tous considèrent les comptes clients

Corine Eyraud. Sociologie du Travail, 2003, n 4, pp. 491-508. 
comme des prêts, et font peu de différence entre une subvention, un prêt bancaire et un investissement de l'État ${ }^{17}$. L'idée que tout cet argent provient de la même poche, de la «grande marmite commune », cette vision totalisante ou moniste est encore très prégnante. C'est ce qu'exprime un directeur d'entreprise : «Nous devons de l'argent à des entreprises d'État qui nous doivent de l'argent, nous devons de l'argent aux banques d'État, nous devons de l'argent à l'État, nous sommes une entreprise d'État. Pour moi, c'est absurde, c'est le Parti communiste qui doit au Parti communiste ».

Cela dit, la réforme comptable semble participer au développement de l'esprit de calcul; cela n'a rien de bien étonnant, de nombreux historiens et sociologues ont montré que le développement de la comptabilité d'entreprise a accompagné le développement de la rationalité économique et du capitalisme (voir par exemple : Braudel, 1979; Sombard, 1932 ; Weber, 1971, 1991) ${ }^{18}$. Cet aspect apparaît clairement dans un des entretiens de directeur

${ }^{17}$ Le directeur d'une agence bancaire française installée en Chine nous signalait d'ailleurs que les directeurs d'entreprises chinoises venaient presque toujours le trouver en lui demandant d'investir dans leur entreprise, et lui s'évertuait à leur expliquer qu'il n'investissait pas mais qu'il prêtait de l'argent. D'un autre côté, la difficulté de différencier les subventions et les investissements de l'État ne semble pas particulière aux entreprises d'État chinoises. Le PDG d'une entreprise publique française interrogé pour une émission radiophonique de France Inter («Rue des entrepreneurs ») en octobre 1996, déclarait ainsi à l'antenne " il est toujours difficile de faire la différence entre une subvention et une augmentation de capital ».

${ }^{18}$ Ceci dit, ces auteurs, et d'autres ultérieurs, ne s'entendent ni sur la place ni sur la force de la comptabilité dans ce processus. Sombart et Weber y accorde une place prépondérante, Braudel une place parmi d'autres (la lettre de change aurait eu d'après lui une force supérieure). Goody estime également qu'elle est «une composante parmi d'autres dans l'élaboration progressive d'un système complexe de production et d'échange » (1999, p.104), et réfute, en s'appuyant sur l'histoire du commerce et de la comptabilité au Proche-Orient et en Asie (Chine et Inde), l'idée wéberienne selon laquelle la rationalité occidentale et la comptabilité en partie double seraient intrinsèquement liées au capitalisme, en seraient des conditions de possibilité. Pour Goody, les concepts abstraits de profit, de firme, d'évaluation du capital seraient moins indissociablement liés au développement de l'économie industrielle moderne, qu'intrinsèques aux toutes premières grandes entreprises commerciales en Asie comme en Europe (1999, chap.2: «rationalité et ragioneria : la tenue des livres de comptes et le miracle économique », pp. 66-106).

Corine Eyraud. Sociologie du Travail, 2003, n 4, pp. 491-508. 
d'entreprise : «Avec cette nouvelle comptabilité, les profits apparaissent clairement, je vois comment les augmenter : il faut compter; j'ai besoin de tel matériau, à tel endroit il est à 9600 yuan la tonne, à tel autre à 10000 yuan, le premier représente une économie, il faut que nous ayons un esprit de calcul; de la même manière il faut diminuer les gaspillages en matières premières et en produits finis ».

L'utilisation des bilans dans la décision de prêts bancaires entre, quant à elle, en concurrence, si ce n'est en conflit, avec d'autres pratiques, que sont les prêts pré-affectés, les pressions effectuées par le gouvernement ou le comité du Parti locaux, la pression interne à la banque qui est de conserver du travail à ses employés, et finalement la dimension relationnelle ; ces différents aspects étant largement imbriqués.

Les prêts pré-affectés sont appelés, en chinois, des «prêts conformément aux ordres » ou des «prêts fixés en fonction de politiques ». Voici ce que nous en dit une personne travaillant au service des prêts d'une agence bancaire pékinoise : «Les "prêts fixés en fonction de politiques" sont particulièrement nombreux, ce sont des prêts dont la direction est fixée : cette entreprise ne peut plus payer ses dépenses, les échelons supérieurs nous envoient un document nous demandant de lui donner un prêt de tant, rien dans la tête seulement un ordre, cet argent c'est sûr qu'il ne reviendra pas mais il faut lui donner le prêt. Parfois, le document descend de notre succursale, parfois du comité du Parti de Pékin, ou encore de l'État. Pour décider de ces prêts, ce ne sont pas les profits économiques qui importent, mais

Corine Eyraud. Sociologie du Travail, 2003, n 4, pp. 491-508. 
les points de vue administratif et politique ${ }^{19}$. La pression du gouvernement ou du comité du Parti locaux est particulièrement forte quand les difficultés de l'entreprise lui empêchent de payer les salaires, dans le souci d'éviter les troubles sociaux.

L'octroi de prêts peut également être une quasi-nécessité pour la survie même de la banque : « Il y a des entreprises en situation difficile dans lesquelles je n'aime pas descendre. Comme le Groupe X qui est en pleine crise. Nous devons lui accorder des prêts parce que les relations entre lui et nous sont à la vie à la mort : s'il fait faillite, notre banque n'ira pas bien non plus. Parce que chez lui, nous avons une agence de cent personnes, qui a été ouverte pour lui ; la plupart des activités de cette agence, ce sont les activités de ce groupe, ses paiements et ceux des usines auxiliaires et des sociétés qu'il a créées. Aussi, nous devons trouver des moyens pour lui accorder des prêts ; si on le peut on le doit, parce que, s'il est compétitif, alors les cent personnes de notre agence ont du travail et peuvent même créer des profits ».

Finalement, écoutons un expert-comptable : «En Chine, quand je veux emprunter de l'argent à une banque, j'utilise les relations interpersonnelles (guanxi). Je suis chef comptable d'une entreprise, tu travailles dans une banque, si nos relations sont bonnes, nous sommes des amis, avant nous étions à la même

\footnotetext{
${ }^{19}$ Les grandes banques d'État sont ainsi confrontées à des logiques clairement contradictoires : le gouvernement, au travers des réformes, leur demande d'agir en tant qu'entités économiques responsables de leurs profits et de leurs pertes et donc d'octroyer des prêts sur des critères économiques, et en même temps leur ordonne d'octroyer un prêt à telle ou telle entreprise en difficulté.
}

Corine Eyraud. Sociologie du Travail, 2003, n 4, pp. 491-508. 
école ou dans la même unité de travail ${ }^{20}$, peu importe ma situation financière, je te donne un peu d'argent ou d'autres avantages, et tu n'as pas peur puisque la banque n'est pas à toi. Alors tu n'attaches pas tellement d'importance aux documents financiers. J'ai entendu tellement de chefs comptables parler, quelle est ta situation financière, de combien sont tes profits, peu importe, tu as des amis à la banque ça suffit ».

Ces différents aspects sont renforcés par le fait que la demande en prêts bancaires excède largement 1 'offre ${ }^{21}$. Quant aux décisions gouvernementales de mise en faillite d'entreprises d'État, malgré l'adoption d'une loi sur les faillites en 1988, elles sont quasiment inexistantes avant 1997.

Finalement, on peut dire que l'efficacité sociale de la réforme comptable était, trois ans après sa mise en route, relativement limitée. La nouvelle comptabilité ne semblait pas avoir rencontré dans l'état des structures économiques et sociales chinoises les moyens de se rendre efficace.

\subsubsection{Fin des années 1990 - Début des années 2000}

La dissociation des catégories économico-comptables est, dans les discours, largement plus prononcée que pendant la période précédente; le terme de «prêts », par exemple, n'est plus utilisé pour parler des créances clients mais uniquement pour parler de prêts bancaires. Cette dissociation n'est toutefois pas

\footnotetext{
${ }^{20}$ L'unité de travail ou «danwei » est le nom donné en Chine à toute organisation : un ministère, une entreprise, un hôpital, une école, etc.

${ }^{21}$ Cela dit, ces différentes pratiques ne sont pas spécifiques à la situation chinoise. L'affaire du Crédit Lyonnais ou celle d'Enron sont là pour nous le rappeler : «Les banques d'affaire sont impliquées dans l'affaire Enron (...), de peur de perdre un client et leur intérêts déjà investis, elles ont préféré se taire, voire même conseiller les titres à l'achat par l'intermédiaire de leurs analystes financiers, profession elle aussi sérieusement discréditée par le scandale Enron.» (Ferrandon, 2002, p.69).
}

Corine Eyraud. Sociologie du Travail, 2003, n 4, pp. 491-508. 
aboutie, un directeur d'entreprise nous signale : «Une banque est venue investir dans notre entreprise », et répond à notre demande d'explicitation (« investir ou prêter de l'argent ?») : «C'est pareil, en fait elle nous a donné un prêt ». Cette dissociation n'est pas facilitée par certaines réformes récentes, en particulier celle consistant à «transformer des dettes en actions » (zhai gai gu) ou, autre appellation, à «transformer des dettes en investissements »(zhai gai tou). Les entreprises d'État étant largement surendettées et donc accablées de frais financiers, cette réforme vise à transformer une partie de leurs prêts en actions sur leur capital détenues par les banques concernées. Là encore, la nouvelle comptabilité ne semble pas rencontrer dans l'état des structures économiques et sociales les moyens de se rendre efficace.

Les mêmes remarques peuvent être faites à propos des prêts bancaires. La tendance générale est à l'octroi de prêts sur la base de critères économiques et donc à la lumière de l'analyse des états financiers de l'entreprise. Cette tendance est fortement et régulièrement réaffirmée par le premier ministre chinois de l'époque, Zhu Rongji, des sanctions sont prises contre des dirigeants d'agences ou succursales bancaires n'allant pas dans ce sens et différentes réformes organisationnelles tentent de couper le lien entre banques et gouvernements au niveau local. On peut ainsi penser que l'évolution ne se produit pas aussi vite que le souhaiterait le premier ministre, vus la réitération de ces discours et la publicité faite autour des sanctions.

En revanche, là où cette réforme est d'une efficacité féroce c'est, depuis 1997, dans la mise en faillite et les plans de licenciement. Le président de

Corine Eyraud. Sociologie du Travail, 2003, n 4, pp. 491-508. 
l'époque, Jiang Zemin, dans son discours au XVe congrès du Parti en septembre 1997, prend une position tranchée sur la question des sureffectifs des entreprises d'État, et déclare qu'il faut «choisir une réduction du personnel pour une augmentation des résultats ». Le chef du gouvernement, Zhu Rongji, annonce, quant à lui, que le problème des pertes des entreprises d'État doit être réglé dans les trois ans et menace de démissionner si ce n'est pas le cas. Une pression énorme sur les gouvernements locaux s'ensuit ; une grande campagne d'audit est lancée, d'analyse des bilans des entreprises d'État, de repérage de celles connaissant des pertes importantes et chroniques, d'autorisations à partir de ce repérage de plans de licenciement et de pré-retraites, et de mise en faillite. Le bilan, quoique difficile à chiffrer précisément, est considérable. La réduction de personnel ou la gestion des personnels d'entreprises mises en faillite se fait à travers l'invention d'une nouvelle catégorie de sans-travail: les xiagang (littéralement ceux qui sont descendus de poste, ou autrement dit ceux qui n'ont plus de poste). Sur l'ensemble de la période 1994-2000, en sachant que le gonflement de cette catégorie se réalise essentiellement de 1997 à 2000, on estime qu'aurait été « descendus de poste » de 36 à 55 millions de personnes, soit de un tiers à la moitié des employés d'entreprises d'État répertoriés en 1994, ou de 20 à $30 \%$ de l'emploi urbain de cette même année ${ }^{22}$ ! Ces estimations ne rendent pas complètement compte de l'ampleur du phénomène, en particulier elles laissent de côté la pratique de la mise en retraite anticipée: parfois dès 40 ans pour les

22 Ces estimations proviennent de : J.L Rocca, 2000, La montée du chômage dans la Chine urbaine, Perspectives chinoises 59, 38-51. J.-L. Rocca y explique les incertitudes de ces statistiques (d'où une fourchette aussi importante), et les modalités de gestion sociale de cette catégorie. Le taux de réemploi de cette catégorie varie selon les sources de 20 à $70 \%$.

Corine Eyraud. Sociologie du Travail, 2003, n 4, pp. 491-508. 
femmes, 50 ans pour les hommes, et avec un salaire très réduit.

\section{Comptabilité et sociologie}

A partir de cette analyse de la réforme comptable chinoise, nous pouvons dégager différents éléments d'ordre général. Premièrement, il nous semble clair que la comptabilité est l'objet d'enjeux sociaux, de négociations et de luttes entre les acteurs intéressés (entre par exemple les ministères industriels chinois et le Bureau national des Statistiques); ses règles, catégories et documents sont finalement le résultat du rapport des forces en présence. C'est ce que nous a montré l'analyse de l'élaboration des systèmes comptables et financiers chinois, c'est également ce que pourrait montrer l'analyse du processus d'harmonisation des normes comptables européennes ou plus largement internationales. Deuxièmement, la comptabilité véhicule des modèles de représentation de la réalité, en particulier des modèles de représentation de ce qu'est et de ce que n'est pas une entreprise, de ce que sont et ne sont pas ses objectifs. Troisièmement, la comptabilité n'est pas seulement un produit de l'histoire mais elle est aussi productrice d'histoire, dans le sens où elle participe à la structuration des pratiques économiques et des anticipations des acteurs. Finalement, il existe une très forte cohérence, on pourrait même parler d'unité, entre système comptable forme d'entreprise - système de fonctionnement économique et mode de gestion du social. Et il n'est pas anodin que la France, pays où l'État intervient largement dans le domaine des activités économiques, possède un plan comptable appliqué obligatoirement par toutes les entreprises et élaboré par le Conseil National de la

Corine Eyraud. Sociologie du Travail, 2003, n 4, pp. 491-508. 
Comptabilité qui compte plusieurs représentants de l'État (en particulier du ministère des Finances) ; alors que les États-Unis ne possèdent que des normes comptables générales élaborées par le Financial Accounting Standards Board composé exclusivement de professionnels de la comptabilité, normes à partir desquelles chaque entreprise construit son propre plan comptable.

L'objectif de notre démonstration était de remettre en cause l'illusion objectiviste portée par la comptabilité ${ }^{23}$. La comptabilité est, pour la sociologie, non seulement un objet possible et légitime, mais également une entrée intéressante pour l'analyse de phénomènes et processus plus larges ${ }^{24}$.

\section{Références}

Braudel, F., 1979, Civilisation matérielle, économie et capitalisme, $15^{\mathrm{e}}-18^{\mathrm{e}}$ siècle, A. Colin, Paris, Tome 2.

Boyer, R., 1993, La grande transformation à l'Est: une lecture régulationniste, CEPREMAP, Working Paper (couverture orange).

Callon, M., 1999, La sociologie peut-elle enrichir l'analyse économique des externalités ? Essai sur la notion de cadrage-débordement. In : Foray et Mairesse (Ed.), Innovations et performances, Editions de l'EHESS, Paris, 399-431.

${ }^{23}$ En fait, cette illusion objectiviste est plus portée par l'image communément admise de la comptabilité, que par les praticiens ou les penseurs de la comptabilité eux-mêmes. Nombre d'entre eux mènent une réflexion qui va tout à fait dans notre sens (cf. Capron, 1985, ou Colasse (dir.), 2000).

${ }^{24}$ Un numéro de la revue «Comptabilité, Contrôle, Audit » (revue de l'Association francophone de Comptabilité) va être consacré à «La sociologie de la comptabilité »; coordonné par Eve Chiapello, il devrait être publié au début de l'année 2004, et permettre de nourrir la réflexion amorcée ici.

Corine Eyraud. Sociologie du Travail, 2003, n 4, pp. 491-508. 
Capron, M., 1985, La Comptabilité, La Découverte, Paris.

Capron, M., Leseul, G., 1997, Pour un bilan sociétal des entreprises, Revue des études coopératives mutualistes et associatives (RECMA) 266, 28-41.

Centre des jeunes dirigeants d'entreprise (CJD), 1996, L'entreprise au XXI siècle, Flammarion, Paris.

Colasse, B. (dir.), 2000, Encyclopédie de comptabilité, contrôle de gestion et audit, Economica, Paris.

Donzelot, J., 1984, L'Invention du social, essai sur le déclin des passions politiques, Fayard, Paris.

Eyraud, C., 1999, L'entreprise d'État chinoise : de "l'institution sociale totale" vers l'entité économique ?, L’Harmattan, Paris.

Ferrandon, B., 2002, Les leçons de l'affaire Enron, Les Cahiers Français 309, 69.

Friedberg, E., Musselin, C., Les externalités d'un point de vue sociologique. Quelques remarques. In : Foray et Mairesse (Ed.), Innovations et performances, Editions de l'EHESS, Paris, 447-451.

Goody, J., 1999, L’Orient en Occident, Seuil, Paris.

Laville, J.-L., 1997, Le renouveau de la sociologie économique, Cahiers internationaux de Sociologie CIII, 229-235.

Mangenot, M., 1976, La comptabilité au service du capital, Jean-Pierre Delarge éditeur, Paris.

North, D.C., 1990, Institutions, institutional change and economic performance,

Corine Eyraud. Sociologie du Travail, 2003, n 4, pp. 491-508. 
Cambridge University Press, Cambridge.

Salais, R., Baverez, N., Reynaud, B., 1986, L’invention du chômage, PUF, Paris.

Shanghai University of Finance and Economics and University of Texas at Dallas, 1987, Accounting and Auditing in the People's Republic of China, Shanghai University Press, Shanghai.

Sombard, W., 1932, L’apogée du capitalisme, Payot, Paris.

Tang (dir.), 1994, Accounting and Finance in China, a Review of Current Practice, Longman Group, Hong Kong.

Thévenot, L., 1985, Les investissements de forme, Cahiers du centre d'études de l'emploi 29, 21-71.

Vercueil, J., 2001, Transition et ouverture de l'économie russe (1992-2002) Pour une économie institutionnelle du changement, L'Harmattan, Paris.

Weber, M., 1971, Economie et sociétés, Plon, Paris.

Weber, M., 1991, Histoire économique - Esquisse d'une histoire universelle de l'économie et de la société, Gallimard, Paris.

Corine Eyraud. Sociologie du Travail, 2003, n 4, pp. 491-508. 